\title{
Association of elevated risk of pancreatic cancer in diabetic patients: A systematic review and meta-analysis
}

\author{
JIAXIN TAN ${ }^{1}$, YU YOU ${ }^{1}$, FEI GUO ${ }^{1}$, JIANHUA XU ${ }^{2}$, HAISU DAI $^{1}$ and PING BIE ${ }^{1}$ \\ ${ }^{1}$ Department of Hepatobiliary Surgery, The First Affiliated Hospital, The Third Military Medical University, \\ Chongqing 400038; ${ }^{2}$ Department of Hepatobiliary Surgery, Daping Hospital and Research Institute of Surgery, \\ The Third Military Medical University, Chongqing 400042, P.R. China
}

Received July 7, 2016; Accepted January 4, 2017

DOI: $10.3892 / \mathrm{ol} .2017 .5586$

\begin{abstract}
Pancreatic cancer has a five-year overall survival rate $<5 \%$, a situation that has not improved since for 40 years. Diabetes mellitus including type 2 diabetes mellitus (T2D) is a suspected risk factor for the development of pancreatic cancer and nearly $45 \%$ of the pancreatic cancer cases are likely to present as new onset diabetes cases; however, the nature of association between T2D and pancreatic cancer is still controversial. In this meta-analysis, we examined the association specifically of T2D with pancreatic cancer and the influence of insulin therapy. PubMed, EMBASE, Scholar, Web of Science and Scopus databases were searched to identify clinical and patient oriented studies that examined the incidence of diabetes in pancreatic cancer patients and vice versa, over the last 10 years. All the authors independently screened the articles, and a collective decision was reached about the studies included in the meta-analysis. Parameters analyzed included, the Incidence of diabetes in pancreatic cancer patients; duration history of T2D in pancreatic cancer patients; influence of insulin therapy in T2D patients on pancreatic cancer incidence. Eleven studies with a total of 14,399 patients, of whom 4,080 were T2D-positive and 9,721 were non-diabetic were included in this meta-analysis. T2D duration history was significantly related to pancreatic cancer incidence and insulin therapy effects. In conclusion, recent-onset T2D is probably a manifestation of pancreatic cancer whereas long-term T2D is likely a risk factor for this cancer. Insulin therapy appears to decrease the incidence of pancreatic cancer.
\end{abstract}

\section{Introduction}

Pancreatic cancer has one of the highest mortality rates in cancer, with five-year overall survival (OS) rate $<5 \%$

Correspondence to: Dr Ping Bie, Department of Hepatobiliary Surgery, The First Affiliated Hospital, The Third Military Medical University, 30 Gaotanyan Street, Chongqing 400038, P.R. China E-mail: bie_ping1@163.com

Key words: pancreatic cancer, type 2 diabetes, insulin therapy, carbohydrate antigen 19-9, overall survival, HbAlc and despite significant advances in medical technologies, this situation of short survival has not improved for four decades. The dismal prognosis of pancreatic cancer patients and the lack of effective drugs to improve survival beyond few months, necessitates understanding of the pathogenic mechanisms of this cancer and the factors that drive its rapid growth and to identify better and efficacious therapeutics. It has been thought that diabetes mellitus is a primary risk factor for the development of pancreatic cancer and nearly $45 \%$ of the pancreatic cancer cases are likely to present as new onset diabetes cases, with somewhat elevated incidence in males $(1,2)$. The strong link between diabetes and pancreatic cancer can be mostly due to new-onset diabetes. However, whether diabetes is a risk factor for pancreatic cancer or only the manifestation of pancreatic cancer has been debated and more studies suggested that diabetes as well as metabolic syndrome are potential risk factors for pancreatic cancer (3). Type 2 diabetes mellitus (T2D) is a metabolic disease with characteristic hyperglycemia that arises because of defective insulin secretion by pancreatic islets in response to elevated post-prandial blood glucose levels and insulin resistance of peripheral tissues as well as dysregulated hepatic glucose production (4). T2D is often associated with obesity, which has been on the increase across the globe. Defective response of body tissues to insulin, i.e., reduced insulin sensitivity, places an increased demand on pancreatic islets to secrete higher quantities of insulin, leading to hyperinsulinemia and these are usually the primary events in the pathogenesis of T2D, which are precipitated by genetic as well as environmental factors (5).

Insulin is known to function as a regulator of glucose and lipid metabolism and to act as a potential growth factor in the promotion of cell proliferation and angiogenesis. Although diabetes and obesity are suspected of increasing the risk of different types of cancers, the risk for pancreatic cancer is thought to be particularly high (6). Malignant transformation of pancreatic cells, predominantly the ductal epithelial cells of the exocrine pancreas, when associated with locally elevated supply of insulin coming from the endocrine islets of pancreas, grow rapidly and probably such an association is the cause for the devastating outcome of pancreatic cancer (7). The nature of association between T2D and pancreatic cancer is controversial as some studies indicated T2D lowering the OS $(8,9)$ 
while others did not find such an effect $(10,11)$. Additionally, epidemiological studies indicated an increased incidence of diabetes in pancreatic cancer and an elevated number of cases of pancreatic cancer in diabetic patients, raising the question whether diabetes is a cause or consequence of pancreatic cancer $(12,13)$. In several of these studies, a clear differentiation between type 1 or 2 diabetes was not made and diabetes incidence was viewed collectively. In the present metaanalysis, we have tried to address this issue of association of T2D with pancreatic cancer, taking into consideration recently published clinical studies with identified T2D patients. Our results suggest that new onset T2D (observed $\leq 2$ years before pancreatic cancer diagnosis) is a strong indicator of pancreatic cancer development and also that the presence of diabetes for $>5$ years can be a significant risk factor for pancreatic cancer.

\section{Materials and methods}

Objectives. In the present meta-analysis, we analyzed the results from clinical studies that examined the co-incidence of T2D and pancreatic adenocarcinoma, along with other variables such as insulin usage as a therapeutic measure. We also analyzed the relationship between the time of diagnosis and history of diabetes and the diagnosis of pancreatic cancer.

\section{Methods}

Criteria for considering studies for this review. Clinical studies that examined the relationship between the incidences of diabetes and pancreatic cancer were included in this meta-analysis. Studies that did not clearly document the diagnosis of diabetes (e.g., by HbAlc levels or hyperinsulinemia) or did not record the patient history of diabetes were excluded. While few studies only examined diabetic patients with pancreatic cancer, others included pancreatic cancer patients without and with a diagnosis of diabetes. In addition, diabetic patients with a history of cancer diagnosis that was not primarily related to pancreatic cancer were excluded from the study. Even though there appears to be a strong male preponderance in the incidence of diabetes in pancreatic cancer patients, we included both male and female patients and analyzed the results collectively, to eliminate any gender-related bias in the study. Detailed selection and exclusion criteria of identified studies for the meta-analysis is shown in Fig. 1.

Search methods. Publications describing the relevant information were searched in PubMed, Google Scholar, Scopus and Web of Science databases. Search MeSH terms included pancreatic cancer, diabetes mellitus, T2D, OS, clinical studies, hyperglycemia, insulin, insulin resistance, pancreatic ductal carcinoma and pancreatic adenocarcinoma. Publications in the last 10 years were searched, as many of the earlier publication did not differentiate between type 1 and T2D in their analysis. All the citations, only in the English language, were initially screened for relevance of the title and abstract level and full reports and supplemental information files were retrieved as per the relevance of the selected study.

Data collection and analysis and quality assessment. All the co-authors of this meta-analysis independently screened the full texts of all the selected studies for the details of

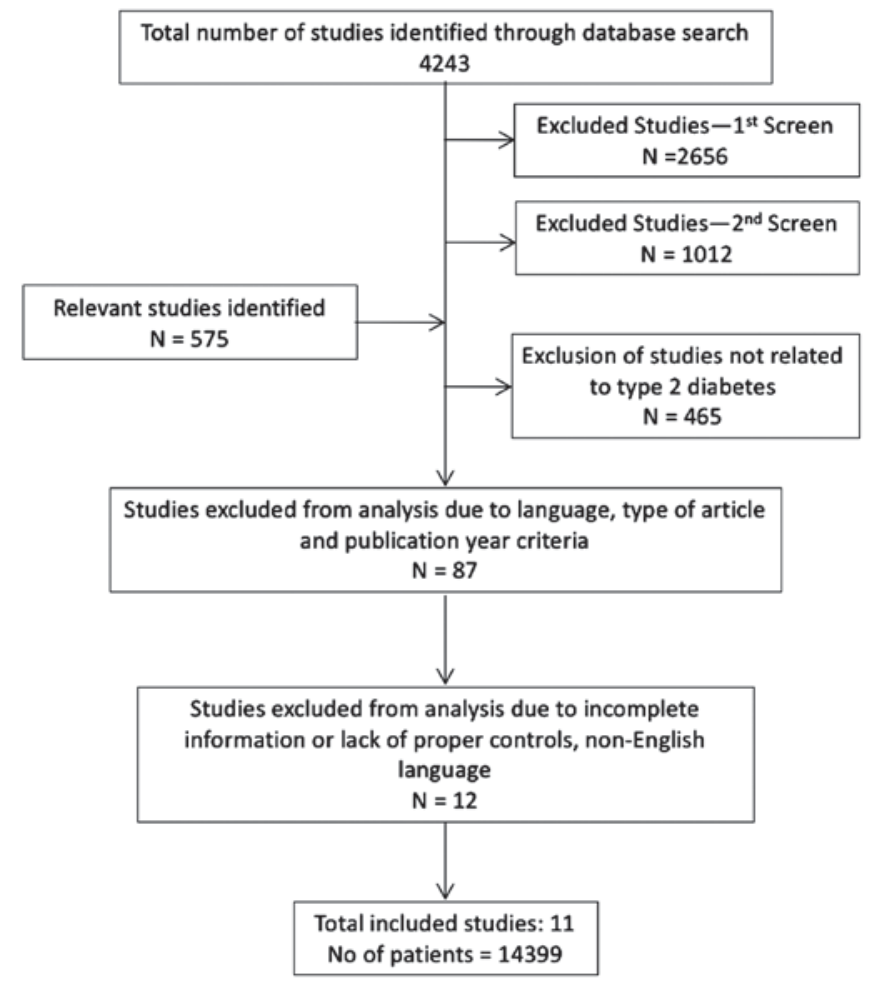

Figure 1. Flow-chart indicating the selection and exclusion of identified studies for the meta-analysis.

patient selection and parameters studied. All the collected information was then pooled and reviewed collectively and any differences of opinion or discrepancies were resolved by discussion and consensus. The following data were extracted from the included studies: Details of the institutions where the studies were conducted and the authors, publication details, the total number of patients studied and the number of patients in pancreatic cancer with T2D group and pancreatic cancer without T2D group, age, and gender of the studied patients. In addition, where available, we collected information regarding the history and onset of diabetes in comparison to the time of diagnosis of pancreatic cancer. OS data were obtained only from a few studies.

Statistical analysis. We prepared a Preferred Reporting Items for Systematic Reviews and Meta-Analyses (PRISMA) checklist (Table I). Methods suggested by the Cochrane Collaboration guidelines for meta-analysis have been used. Statistical analyses were performed by Review Manager (RevMan) version 5.3 (The Cochrane Collaboration). The association of T2D duration before and along with pancreatic cancer was analyzed independent of each other. Mantel-Haenszel statistics were applied in the random-effect model, for the analyses of body mass index (BMI), carbohydrate antigen 19-9 (CA19-9), HbAlc and OS and odds ratios (OR) were derived at $95 \%$ confidence intervals (CI). Mantel-Haenszel statistics were applied in the fixed-effect model for the analyses of association between pancreatic cancer and duration and history of diabetes, and OR were derived at 95\% CI. Publication bias was analyzed by Funnel plot of sample size of each study. Sensitivity analysis was performed for T2D and pancreatic cancer association by excluding one study at a time in analyzing the results. 
Table I. PRISMA check list.

Section/topic

No.

Checklist item

Reported on

page no.

Title

Title

Abstract

Structured summary

Introduction

Rationale

Objectives

Methods

Protocol and registration

Eligibility criteria

Information sources

Search

Study selection

Data collection process

Data items

Risk of bias in

individual studies

Summary measures

Synthesis of results

Risk of bias across studies

Additional analyses

Results

Study selection

Study characteristics

Risk of bias within studies

Results of individual studies

Synthesis of results

Risk of bias across studies

1 Identify the report as a systematic review, meta-analysis, or both.

2 Provide a structured summary including, as applicable: Background; objectives; data sources; study eligibility criteria, participants, and interventions; study appraisal and synthesis methods; results; limitations; conclusions and implications of key findings; systematic review registration number.

3 Describe the rationale for the review in the context of what is already known.

4 Provide an explicit statement of questions being addressed with reference to participants, interventions, comparisons, outcomes, and study design (PICOS). address), and, if available, provide registration information including registration number.

6 Specify study characteristics (e.g., PICOS, length of follow-up) and report characteristics (e.g., years considered, language, publication status) used as criteria for eligibility, giving rationale.

7 Describe all information sources (e.g., databases with dates of coverage, contact with study authors to identify additional studies) in the search and date last searched.

8 Present full electronic search strategy for at least one database, including any limits used, such that it could be repeated.

9 State the process for selecting studies (i.e., screening, eligibility, included in systematic review, and, if applicable, included in the meta-analysis).

10 Describe method of data extraction from reports (e.g., piloted forms, independently, in duplicate) and any processes for obtaining and confirming data from investigators.

11 List and define all variables for which data were sought (e.g., PICOS, funding sources) and any assumptions and simplifications made.

12 Describe methods used for assessing risk of bias of individual studies (including specification of whether this was done at the study or outcome level), and how this information is to be used in any data synthesis.

13 State the principal summary measures (e.g., risk ratio, difference in means).

14 Describe the methods of handling data and combining results of studies, if done, including measures of consistency (e.g., $\mathrm{I}^{2}$ ) for each meta-analysis.

15 Specify any assessment of risk of bias that may affect the cumulative evidence (e.g., publication bias, selective reporting within studies).

16 Describe methods of additional analyses (e.g., sensitivity or subgroup analyses, meta-regression), if done, indicating which were pre-specified.

17 Give numbers of studies screened, assessed for eligibility, and included in the review, with reasons for exclusions at each stage, ideally with a flow diagram.

18 For each study, present characteristics for which data were extracted (e.g., study size, PICOS, follow-up period) and provide the citations.

19 Present data on risk of bias of each study and, if available, any outcome level assessment (see item 12).

20 For all outcomes considered (benefits or harms), present, for each study: a) Simple summary data for each intervention group; b) effect estimates and confidence intervals, ideally with a forest plot.

21 Present results of each meta-analysis done, including confidence intervals and measures of consistency.

22 Present results of any assessment of risk of bias across studies (see item 15). 
Table I. Continued.

\begin{tabular}{|c|c|c|c|}
\hline Section/topic & No. & Checklist item & $\begin{array}{l}\text { Reported on } \\
\text { page no. }\end{array}$ \\
\hline Additional analysis & 23 & $\begin{array}{l}\text { Give results of additional analyses, if done [e.g., sensitivity or subgroup analyses, } \\
\text { meta-regression (see item 16)]. }\end{array}$ & 9 \\
\hline \multicolumn{4}{|l|}{ Discussion } \\
\hline Summary of evidence & 24 & $\begin{array}{l}\text { Summarize the main findings including the strength of evidence for each main } \\
\text { outcome; consider their relevance to key groups (e.g., healthcare providers, users, } \\
\text { and policy makers). }\end{array}$ & 8,9 \\
\hline Limitations & 25 & $\begin{array}{l}\text { Discuss limitations at study and outcome level (e.g., risk of bias), and at review } \\
\text { level (e.g., incomplete retrieval of identified research, reporting bias). }\end{array}$ & 9 \\
\hline Conclusions & 26 & $\begin{array}{l}\text { Provide a general interpretation of the results in the context of other evidence, and } \\
\text { implications for future research. }\end{array}$ & 9 \\
\hline \multicolumn{4}{|l|}{ Funding } \\
\hline Funding & 27 & $\begin{array}{l}\text { Describe sources of funding for the systematic review and other support } \\
\text { (e.g., supply of data); role of funders for the systematic review. }\end{array}$ & N/A \\
\hline
\end{tabular}

PRISMA, Preferred Reporting Items for Systematic Reviews and Meta-Analyses.

Table II. Patient characteristics.

\begin{tabular}{|c|c|c|c|c|c|c|c|}
\hline \multirow[b]{2}{*}{$\begin{array}{l}\text { Study } \\
\text { Author, year (Ref.) }\end{array}$} & \multirow[b]{2}{*}{$\begin{array}{l}\text { Total no. } \\
\text { of cases }\end{array}$} & \multirow[b]{2}{*}{ Males } & \multirow[b]{2}{*}{$\begin{array}{c}\text { Age } \\
\text { (years) }\end{array}$} & \multicolumn{2}{|c|}{$\begin{array}{l}\text { Pancreatic cancer } \\
\text { patients with T2D }\end{array}$} & \multicolumn{2}{|c|}{$\begin{array}{c}\text { Pancreatic cancer patients } \\
\text { without T2D }\end{array}$} \\
\hline & & & & $\begin{array}{c}\text { T2D } \\
\text { patients }\end{array}$ & $\begin{array}{l}\text { No. of patients } \\
\text { with } \mathrm{BMI}>25\end{array}$ & $\begin{array}{l}\text { No. of patients } \\
\text { without T2D }\end{array}$ & $\begin{array}{l}\text { No. of patients } \\
\text { with BMI > } 25\end{array}$ \\
\hline Nakai et al, 2013 (10) & 250 & 147 & 66 & 124 & - & 126 & - \\
\hline Li et al, 2015 (14) & 1,328 & 787 & 67 & 590 & 472 & 738 & 498 \\
\hline Bosetti et al, 2014 (15) & 8,305 & 4,589 & 72 & 1,767 & 1,205 & 6,404 & 3,410 \\
\hline Mizuno et al, 2013 (16) & 40 & 31 & 70 & 40 & - & - & - \\
\hline Li et al, 2011 (17) & 2,192 & 977 & 63 & 448 & 218 & 1,744 & 647 \\
\hline Oberaigner et al, 2014 (18) & 34 & 18 & 66 & 34 & - & - & - \\
\hline Lu et al, 2015 (19) & 529 & 307 & 66 & 175 & 286 & 354 & - \\
\hline Wang et al, 2006 (20) & 532 & 314 & 60 & 68 & 51 & - & - \\
\hline Choi et al, 2016 (21) & 349 & 224 & 60 & 183 & 27 & 166 & 15 \\
\hline Wolpin et al, 2013 (22) & 449 & 128 & 63.1 & 260 & 243 & 189 & - \\
\hline Sadr-Azodi et al, 2015 (23) & 391 & 220 & 67 & 391 & 270 & - & - \\
\hline
\end{tabular}

T2D, type 2 diabetes mellitus; BMI, body mass index.

\section{Results}

Database searches using the MeSH terms yielded a total of 4,243 studies, which also included some non-clinical and experimental studies. First round of screening to eliminate experimental and non-patient studies (a total of 2,656) yielded 1,587 studies. Further screening to eliminate studies that are not clinically oriented (a total of 1,012), resulted in identification of 575 studies. After applying the criteria of T2D inclusion, 465 studies were removed from the analysis. Subsequent screening to remove review type articles and studies older than 10 years, led to exclusion of 87 studies and 23 studies remained in the analysis. Finally, a few more studies were removed due to incomplete information, lack of proper controls and foreign language (other than English) studies, with 11 studies remaining in the final analysis. Inclusion criteria were: Pancreatic cancer diagnosis, history or occurrence of T2D, clear diagnosis of T2D (HbA1c $>6.5 \%)$. Patients with metastatic disease were not excluded from the analysis. Control patients had pancreatic cancer but were not diagnosed with T2D and did not have a history of T2D. The total number of patients in the included studies were 14,399, of which T2D diagnosis was positive in 4,080 patients and the non-diabetics were 9,721. The included studies were (Table II): Nakai et al (2013) (10); Li et al (2015) (14); Bosetti et al (2014) (15); Mizuno et al (2013) (16); Li et al 
Table III. Differences in CA19-9, HbA1c and OS in pancreatic cancer patients with and without T2D.

\begin{tabular}{|c|c|c|c|c|c|c|c|}
\hline \multirow[b]{2}{*}{$\begin{array}{l}\text { Study } \\
\text { Author, year (Ref.) }\end{array}$} & \multirow[b]{2}{*}{$\begin{array}{l}\text { Total no. } \\
\text { of cases }\end{array}$} & \multicolumn{3}{|c|}{ Pancreatic cancer patients with T2D } & \multicolumn{3}{|c|}{ Pancreatic cancer patients without T2D } \\
\hline & & $\begin{array}{l}\text { No. of patients with } \\
\text { CA19.9 (>500) }\end{array}$ & HbA1c, \% & $\begin{array}{c}\text { OS } \\
\text { (months) }\end{array}$ & $\begin{array}{l}\text { No. of patients with } \\
\text { CA19-9 }(>100)\end{array}$ & $\mathrm{HbA} 1 \mathrm{c}, \%$ & $\begin{array}{c}\text { OS } \\
\text { (months) }\end{array}$ \\
\hline Nakai et al, 2013 (10) & 250 & 100 & $8.45+/-0.88$ & $13.3+/-1.1$ & 89 & $5.1+/-0.3$ & $10+/-0.5$ \\
\hline Li et al, 2015 (14) & 1,328 & 335 & - & $11.7+/-1.1$ & 378 & & $14.2+/-1.5$ \\
\hline Bosetti et al, 2014 (15) & 8,305 & - & - & - & & & \\
\hline Mizuno et al, 2013 (16) & 40 & - & - & - & & & \\
\hline Li et al, 2011 (17) & 2,192 & - & - & - & & & \\
\hline Oberaigner et al, 2014 (18) & 34 & - & $7.7+/-0.5$ & - & & & \\
\hline Lu et al, 2015 (19) & 529 & - & $7.9+/-1$ & - & & $6+/-0.2$ & \\
\hline Wang et al, 2006 (20) & 532 & - & - & - & & & \\
\hline Choi et al, 2016 (21) & 349 & 149 & - & $8.4+/-0.8$ & 133 & & $7.5+/-0.6$ \\
\hline Wolpin et al, 2013 (22) & 449 & - & $5.11+/-0.24$ & - & & $5.09+/-0.2$ & \\
\hline Sadr-Azodi et al, 2015 (23) & 391 & - & $7.9+/-1.1$ & - & & & \\
\hline
\end{tabular}

CA19-9, carbohydrate antigen 19-9; OS, overall survival; T2D, type 2 diabetes mellitus.

(2011) (17); Oberaigner et al (2014) (18); Lu et al (2015) (19); Wang et al (2006) (20); Choi et al (2016) (21); Wolpin et al (2013) (22); and Sadr-Azodi et al (2015) (23).

Patient characteristics. The included patients had an average age of 66.5 years, with 6,657 female and 7,742 male patients. A total of 4,080 pancreatic cancer patients with T2D and 9,721 patients without T2D were further analyzed (Table II). Among the T2D pancreatic cancer patients, nearly $68 \%$ were with BMI above $25 \mathrm{~kg} / \mathrm{m}^{2}$ and $47 \%$ of the pancreatic cancer patients without T2D had a BMI above $25 \mathrm{~kg} / \mathrm{m}^{2}$. The average $\mathrm{HbAlc}$ for the diabetic patients is $7.41 \%$, which is above the normal values $(6.5 \%)$ and for non-diabetic patients it is $5.4 \%$. As there is considerable variation in sample size among the included studies, this has introduced significant heterogeneity (see below). The values for CA19-9, a cancer biomarker, are particularly high in T2D pancreatic cancer patients as compared to patients without T2D (Table III). The OS was reported in only three studies and this ranged from 8.4 to 13.3 months in T2D patients with pancreatic cancer and from 7.5 to 14.2 months in pancreatic cancer patients without T2D (Table III).

OR analysis of BMI using Mantel-Haenszel statistics in the random-effect model, revealed that the number of patients having BMI of $<25 \mathrm{~kg} / \mathrm{m}^{2}$ in without T2D group is significantly higher $(\mathrm{P}<0.00001)$ than in group with $\mathrm{T} 2 \mathrm{D}$ pancreatic cancer patients (Fig. 2A). However, a mean difference analysis of HbA1c level between the with and without T2D groups revealed no significant difference (Fig. 2B), which could be because of smaller number of studies and also wide variation, as reflected in significant heterogeneity. Because of this heterogeneity, we examined whether there was any sample size effect bias in the results due to variation in sample size among the included studies, by Funnel plot and found this had introduced significant heterogeneity (Fig. 2C).

OR analysis of CA19-9 cancer biomarker showed that T2D pancreatic cancer patients had a much higher level of CA19-9
$(\mathrm{P}=0.01)$ compared to pancreatic cancer patients without T2D (Fig. 3A). Heterogeneity did not influence the results. No major differences were noted in OS between patients without and with T2D. However, there was significant heterogeneity in the samples (Fig. 3B). We further analyzed the effect of insulin therapy of diabetic patients on the incidence of pancreatic cancer. Results of OR analysis using Mantel-Haenszel statistics in the random-effect model indicated that the incidence of pancreatic cancer is lower $(\mathrm{P}=0.02)$ in $\mathrm{T} 2 \mathrm{D}$ patients on insulin therapy (Fig. 3C).

We compared the influence of diabetes history and duration on the incidence of pancreatic cancer. Because of the relatively smaller number of studies with varying sample size, we performed OR analysis using Mantel-Haenszel statistics in the fixed-effect model. As compared to patients with long history of T2D ( $>5$ years), patients with T2D diagnosed between 2-5 years prior to pancreatic cancer diagnosis had lower $(\mathrm{P}<0.00001)$ incidence of pancreatic cancer (Fig. 4A). However, patients with recent onset of T2D (diagnosed $<2$ years before cancer detection) had higher $(\mathrm{P}<0.00001)$ incidence of pancreatic cancer (Fig. 4B). Comparison of T2D patients with diagnosis 2-5 years before cancer detection with recent onset T2D patients ( $<2$ years) revealed that pancreatic cancer incidence is higher $(\mathrm{P}<0.00001)$ in recent onset T2D patients (Fig. 4C). Sensitivity analysis by removing one study at a time, for all the parameters analyzed, revealed no change in the pattern of association of T2D with pancreatic cancer incidence.

\section{Discussion}

Diabetes and pancreatic cancer are associated with each other; however, depending on how the sampling is carried out and the focus of the study, it was not clear whether diabetes promotes pancreatic cancer incidence or diabetes is one of the primary symptoms of developing pancreatic cancer $(12,13)$. Similarly, it has been observed in some studies that diabetes 


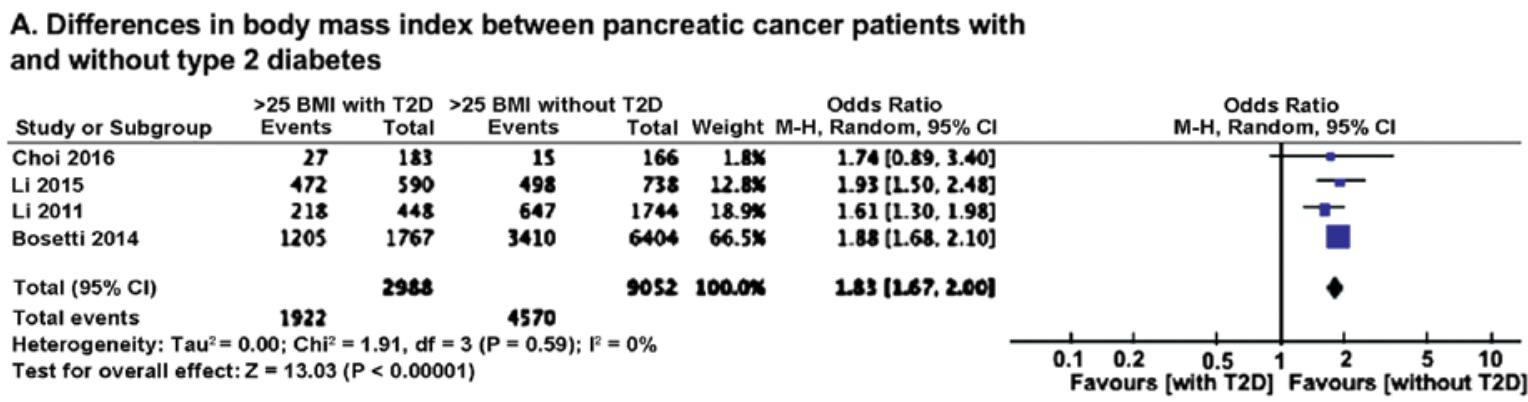

B. Differences in HbA1c between pancreatic cancer patients with and without type 2 diabetes

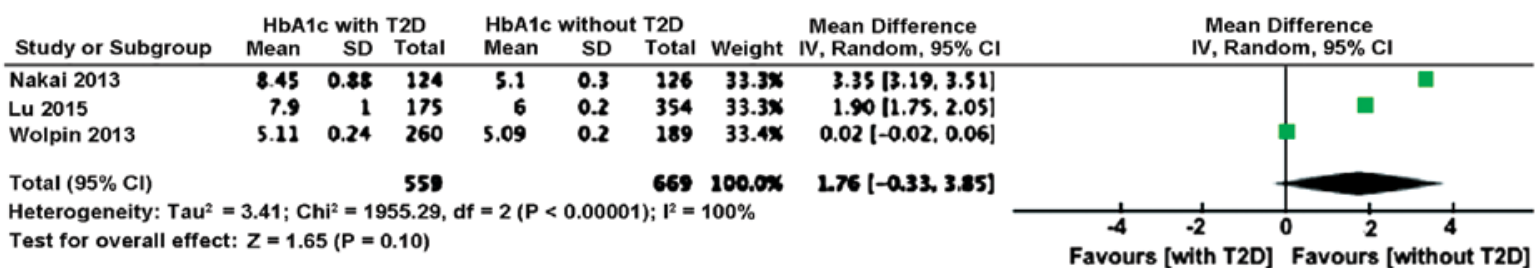

\section{Assessment of bias introduced by sample size in with and without T2D groups in different studies}

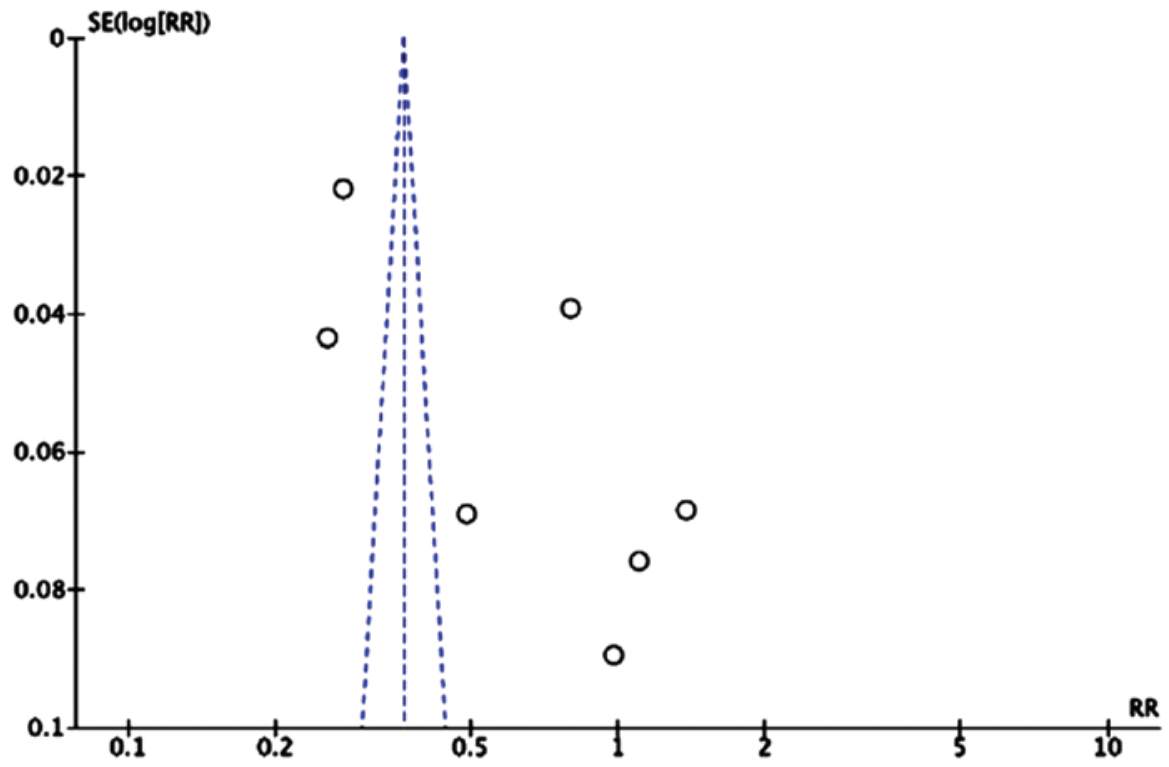

Figure 2. Differences between pancreatic cancer patients with and without T2D and assessment of sample size bias. (A) Differences in BMI; (B) differences in HbAlc; (C) assessment of bias introduced by sample size with and without T2D groups in different studies. T2D, type 2 diabetes mellitus; BMI, body mass index.

has no significant effect on the duration of OS $(10,24)$, but other studies indicated that diabetes significantly reduced the OS $(25,26)$. A recent meta-analysis indicated an association of diabetes with increased overall mortality in pancreatic cancer patients and that the survival of the patient is also dependent on the stage of tumor and the duration of diabetes (27). Also, there is some discrepancy regarding the risk of pancreatic cancer - whether it is insulin resistance or hyperglycemia, both of which are characteristics of T2D. A recent study indicated that peripheral insulin resistance, rather than hyperglycemia or pancreatic $\beta$-cell dysfunction, is associated with risk of pancreatic adenocarcinoma (22).
In the present meta-analysis, we find that the levels of CA19-9 cancer biomarker are much higher in T2D pancreatic cancer patients. Inasmuch as CA19-9 is likely an indicator of the aggressiveness of cancer growth $(28,29)$, the present analysis provides direct evidence showing diabetes promotes rapid growth and aggressiveness of pancreatic cancer and thus likely contributes to increased mortality in these patients. However, because of high level of sample heterogeneity, and smaller number of studies which reported OS, we could not get an accurate estimate of the effect of diabetes on OS.

The present study supports the notion that recent onset diabetes (prior to the diagnosis of pancreatic cancer) is 
A. Differences in CA19-9 cancer biomarker profile between pancreatic cancer patients with and without type 2 diabetes

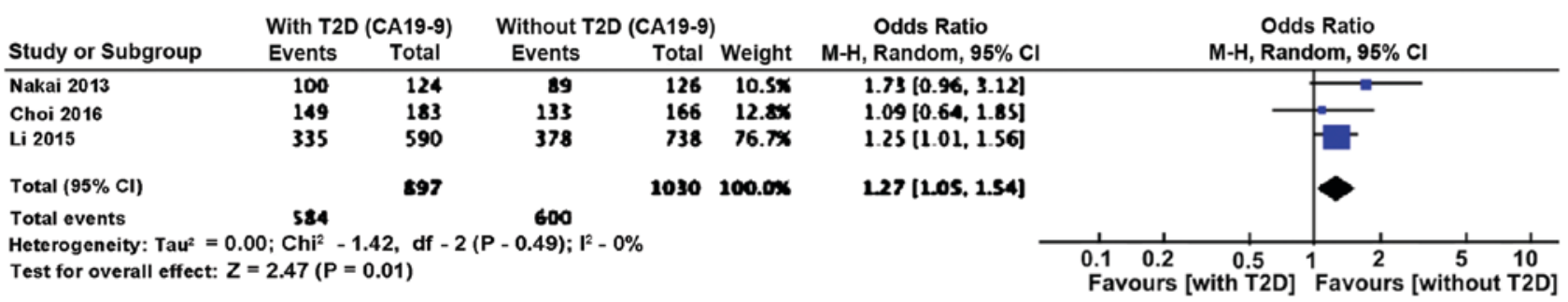

B. Differences in overall survival between pancreatic cancer patients with and without type 2 diabetes

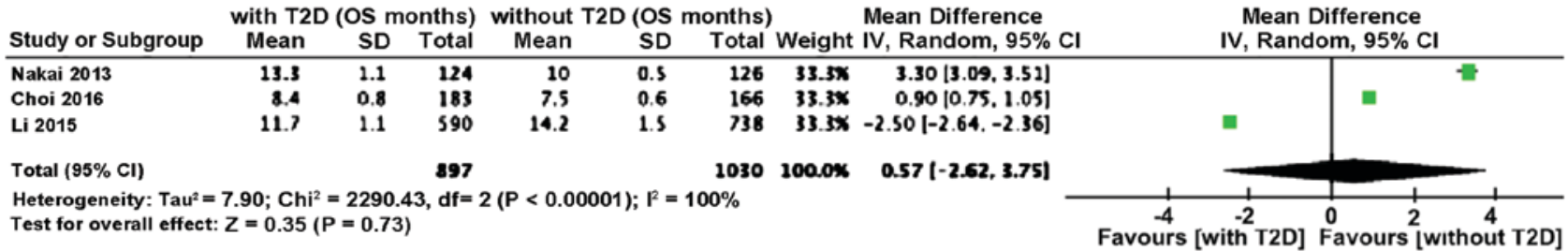

C. Differences in the incidence of pancreatic cancer in type 2 diabetes patients with and without insulin therapy

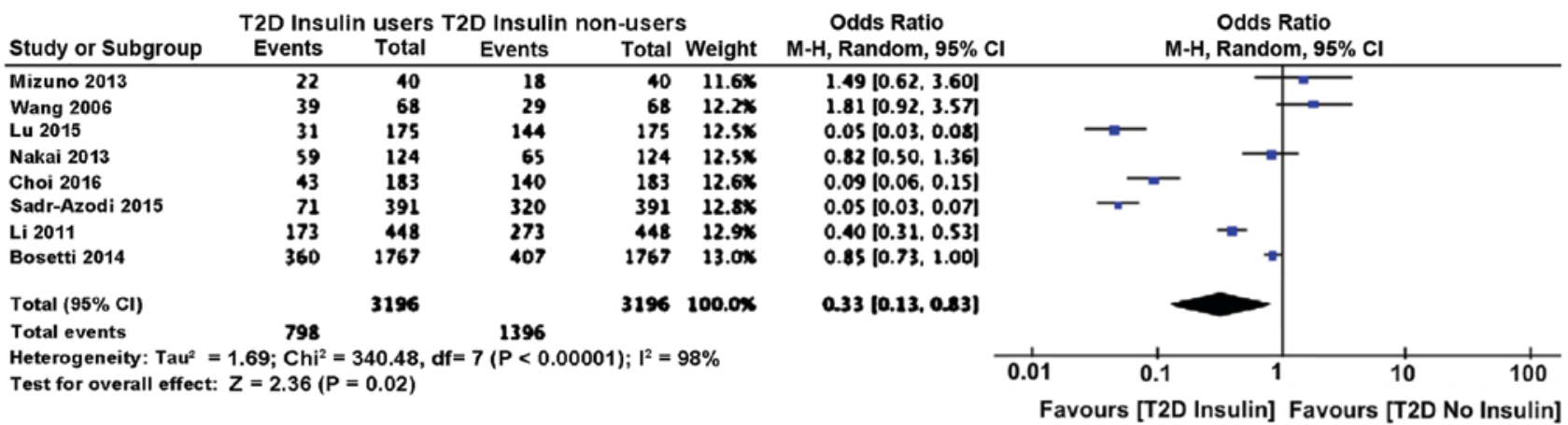

Figure 3. Differences in CA19-9, overall survival and effect of insulin therapy between pancreatic cancer patients with and without T2D. (A) Differences in CA19-9 cancer biomarker profile; (B) differences in overall survival; (C) differences in the incidence of pancreatic cancer in T2D patients with and without insulin therapy. CA19-9, carbohydrate antigen 19-9; T2D, type 2 diabetes mellitus.

significantly associated with increased incidence of pancreatic cancer as compared to 2 years or longer duration of diabetes, prior to pancreatic cancer diagnosis. This is an indication that recent onset diabetes is more likely a manifestation of subclinical pancreatic cancer, rather than a causative or risk factor for cancer. On the other hand, we noted that long-term T2D patients had increased pancreatic cancer incidence, as compared to short-term T2D patients (2-5 years), which is suggestive of the idea that diabetic condition that encompasses insulin resistance and hyperglycemia are likely risk factors for the development of pancreatic cancer, as previously indicated (14). Notably, we found that insulin therapy is associated with reduced pancreatic cancer incidence. As it was indicated and suspected that elevated levels of insulin in pancreas may support the growth of pancreatic tumor (30), it appears that reduced incidence of pancreatic cancer in insulin users is contradictory. However, this finding suggests that while locally produced elevated levels of insulin, which has ready access to the tumor cells of pancreatic cancer and thus promote their aggressive growth, externally administered insulin may hamper or does not promote the development of pancreatic cancer, probably by controlling blood glucose levels and thus reducing the supply of much needed glucose to the pancreatic cancer cells. It is known that pancreatic cancer cells are highly glycolytic and glucose-dependent for their growth $(31,32)$. Therefore, the discrepancy related to the association of long-term diabetes with pancreatic cancer may relate to the proportion of patients who use insulin in a given patient cohort, with higher number of insulin-user patients there may be reduced risk for pancreatic cancer.

Limitations of this study. One major drawback of this meta-analysis is incomplete data for OS of the included patients. Also, not all studies reported HbAlc values for the included T2D patients. Besides this, the included studies suffered from widely varying sample size, which led to high degree of heterogeneity, which probably affected some of the analyses, particularly OS. Another drawback is inclusion of studies, that enrolled only T2D patients with pancreatic cancer, which could have introduced certain degree of bias. 
A. Influence of type 2 diabetes duration on the incidence of pancreatic cancer in patients: comparison of T2D for > 5 years vs. 2 - 5 years.

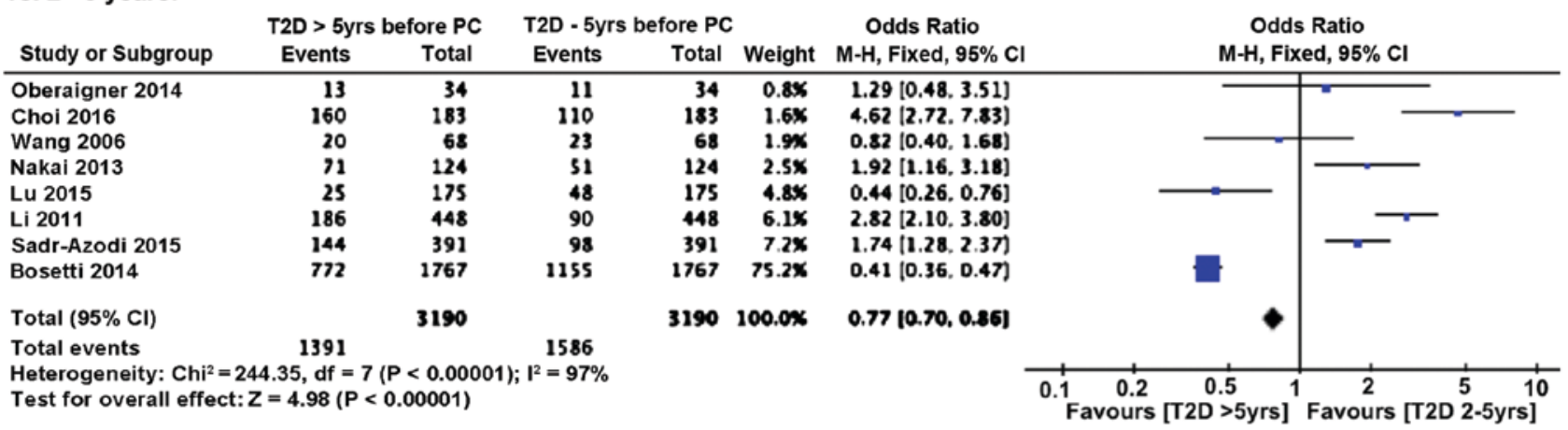

B. Influence of type 2 diabetes duration on the incidence of pancreatic cancer in patients: comparison of T2D for $>5$ years vs. $<2$ years.

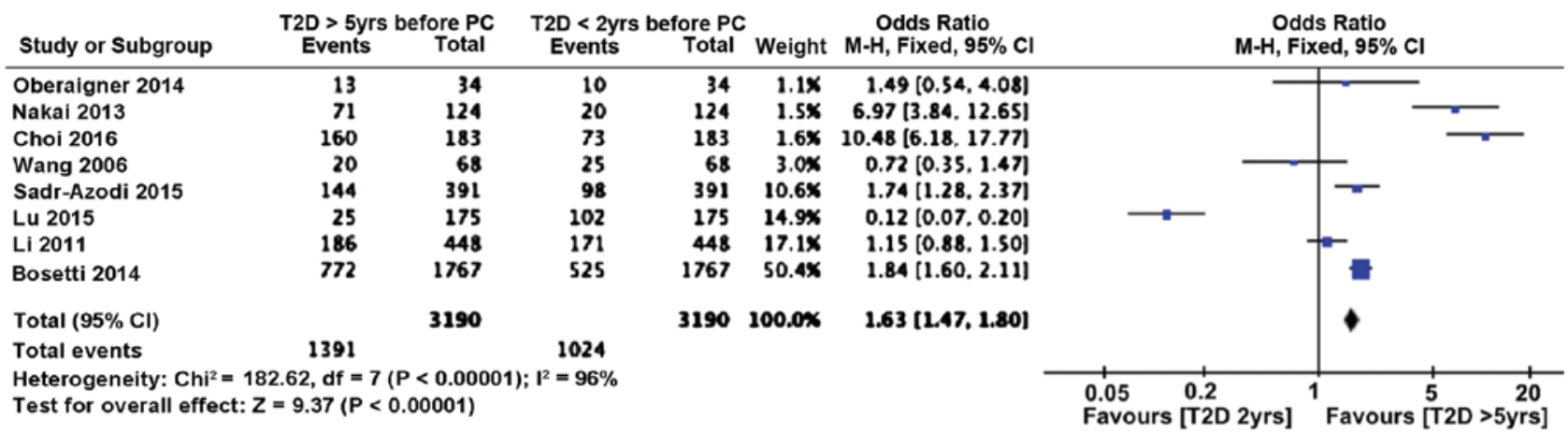

C. Influence of type 2 diabetes duration on the incidence of pancreatic cancer in patients: comparison of T2D for $2-5$ years vs. $<2$ years.

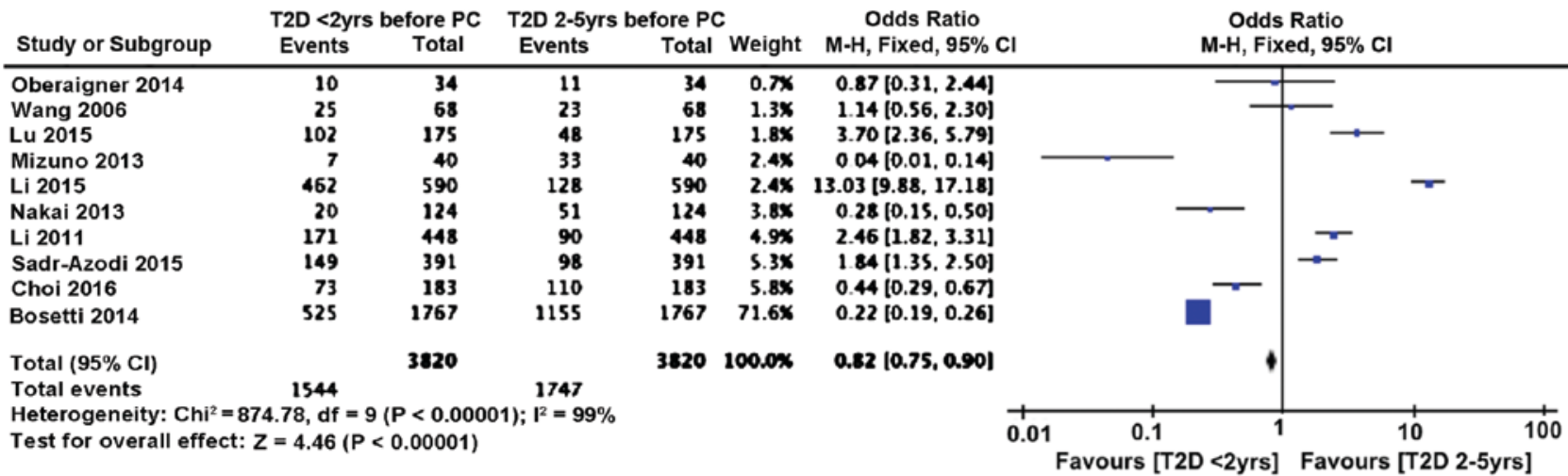

Figure 4. Effect of diabetes history on the incidence of pancreatic cancer. (A) Influence of duration on the incidence of pancreatic cancer in patients: Comparison of T2D for $>5$ vs. $2-5$ years; (B) influence of T2D duration on the incidence of pancreatic cancer in patients: Comparison of T2D for $>5$ vs. $<2$ years. (C) Influence of T2D duration on the incidence of pancreatic cancer in patients: Comparison of T2D for 2-5 vs. $<2$ years. T2D, type 2 diabetes mellitus.

In conclusion, this meta-analysis showed that recent-onset $\mathrm{T} 2 \mathrm{D}$ is probably a manifestation of pancreatic cancer whereas long-term T2D is likely a risk factor for this cancer. The increased mortality of pancreatic cancer patients with T2D is probably related to the aggressive grow th of the tumor, as evidenced by CA19-9 cancer marker. Furthermore, insulin therapy appears to lower the incidence of pancreatic cancer, probably by lowering blood glucose and thus curtailing the supply of glucose to the growing tumor. Further studies are needed to clearly understand the significance of locally produced elevated levels of insulin as compared to the protective effects of externally administered insulin in pancreatic cancer patients with T2D.

\section{References}

1. Ben Q, Cai Q, Li Z, Yuan Y, Ning X, Deng S and Wang K: The relationship between new-onset diabetes mellitus and pancreatic cancer risk: a case-control study. Eur J Cancer 47: 248-254, 2011

2. Jemal A, Siegel R, Ward E, Murray T, Xu J, Smigal C and Thun MJ: Cancer statistics, 2006. CA Cancer J Clin 56: 106-130, 2006.

3. Esposito K, Chiodini P, Colao A, Lenzi A and Giugliano D: Metabolic syndrome and risk of cancer: a systematic review and meta-analysis. Diabetes Care 35: 2402-2411, 2012. 
4. Unger RH and Orci L: Paracrinology of islets and the paracrinopathy of diabetes. Proc Natl Acad Sci USA 107: 16009-16012, 2010.

5. Stumvoll M, Goldstein BJ and van Haeften TW: Type 2 diabetes: principles of pathogenesis and therapy. Lancet 365 : 1333-1346, 2005.

6. Burney S, Irfan K, Saif MW and Masud F: Diabetes and pancreatic cancer. JOP 15: 319-321, 2014.

7. Fokas E, O'NeillE, Gordon-Weeks A, Mukherjee S,McKenna WG and Muschel RJ: Pancreatic ductal adenocarcinoma: from genetics to biology to radiobiology to oncoimmunology and all the way back to the clinic. Biochim Biophys Acta 1855: 61-82, 2015.

8. Walter U, Kohlert T, Rahbari NN, Weitz J and Welsch T: Impact of preoperative diabetes on long-term survival after curative resection of pancreatic adenocarcinoma: a systematic review and meta-analysis. Ann Surg Oncol 21: 1082-1089, 2014.

9. Sahin IH, Shama MA, Tanaka M, Abbruzzese JL, Curley SA, Hassan M and Li D: Association of diabetes and perineural invasion in pancreatic cancer. Cancer Med 1: 357-362, 2012.

10. Nakai Y, Isayama H, Sasaki T, Mizuno S, Sasahira N, Kogure H, Kawakubo K, Yamamoto N, Hirano K, Ijichi H, et al: Clinical outcomes of chemotherapy for diabetic and nondiabetic patients with pancreatic cancer: better prognosis with statin use in diabetic patients. Pancreas 42: 202-208, 2013.

11. Vickers MM, Powell ED, Asmis TR, Jonker DJ, Hilton JF, O'Callaghan CJ, Tu D, Parulekar W and Moore MJ: Comorbidity, age and overall survival in patients with advanced pancreatic cancer - results from NCIC CTG PA.3: a phase III trial of gemcitabine plus erlotinib or placebo. Eur J Cancer 48 : $1434-1442,2012$

12. Chari ST, Leibson CL, Rabe KG, Timmons LJ, Ransom J, de Andrade $M$ and Petersen GM: Pancreatic cancer-associated diabetes mellitus: prevalence and temporal association with diagnosis of cancer. Gastroenterology 134: 95-101, 2008.

13. Li J, Cao G, Ma Q, Liu H, Li W and Han L: The bidirectional interation between pancreatic cancer and diabetes. World J Surg Oncol 10: 171, 2012 .

14. Li D, Mao Y, Chang P, Liu C, Hassan MM, Yeung SJ and Abbruzzese JL: Impacts of new-onset and long-term diabetes on clinical outcome of pancreatic cancer. Am J Cancer Res 5: 3260-3269, 2015

15. Bosetti C, Rosato V, Li D, Silverman D, Petersen GM, Bracci PM Neale RE, Muscat J, Anderson K, Gallinger S, et al: Diabetes, antidiabetic medications, and pancreatic cancer risk: an analysis from the International Pancreatic Cancer Case-Control Consortium. Ann Oncol 25: 2065-2072, 2014.

16. Mizuno S, Nakai Y, Isayama H, Yanai A, Takahara N, Miyabayashi K, Yamamoto K, Kawakubo K, Mohri D, Kogure H, et al: Risk factors and early signs of pancreatic cancer in diabetes: screening strategy based on diabetes onset age. J Gastroenterol 48: 238-246, 2013.

17. Li D, Tang H, Hassan MM, Holly EA, Bracci PM and Silverman DT: Diabetes and risk of pancreatic cancer: a pooled analysis of three large case-control studies. Cancer Causes Control 22: 189-197, 2011.

18. Oberaigner W, Ebenbichler C, Oberaigner K, Juchum M, Schönherr HR and Lechleitner M: Increased cancer incidence risk in type 2 diabetes mellitus: results from a cohort study in Tyrol/Austria. BMC Public Health 14: 1058, 2014.
19. Lu Y, García Rodríguez LA, Malgerud L, González-Pérez A, Martín-Pérez M, Lagergren J and Bexelius TS: New-onset type 2 diabetes, elevated $\mathrm{HbAlc}$, anti-diabetic medications, and risk of pancreatic cancer. Br J Cancer 113: 1607-1614, 2015.

20. Wang F, Gupta S and Holly EA: Diabetes mellitus and pancreatic cancer in a population-based case-control study in the San Francisco Bay Area, California. Cancer Epidemiol Biomarkers Prev 15: 1458-1463, 2006.

21. Choi Y, Kim TY, Oh DY, Lee KH, Han SW, Im SA, Kim TY and Bang YJ: The impact of diabetes mellitus and metformin treatment on survival of patients with advanced pancreatic cancer undergoing chemotherapy. Cancer Res Treat 48: 171-179, 2016.

22. Wolpin BM, Bao Y, Qian ZR, Wu C, Kraft P, Ogino S, Stampfer MJ, Sato K, Ma J, Buring JE, et al: Hyperglycemia, insulin resistance, impaired pancreatic $\beta$-cell function, and risk of pancreatic cancer. J Natl Cancer Inst 105: 1027-1035, 2013.

23. Sadr-Azodi O, Gudbjörnsdottir S and Ljung R: Pattern of increasing $\mathrm{HbA} 1 \mathrm{c}$ levels in patients with diabetes mellitus before clinical detection of pancreatic cancer - a populationbased nationwide case-control study. Acta Oncol 54: 986-992, 2015.

24. Dandona M, Linehan D, Hawkins W, Strasberg S, Gao F and Wang-Gillam A: Influence of obesity and other risk factors on survival outcomes in patients undergoing pancreaticoduodenectomy for pancreatic cancer. Pancreas 40: 931-937, 2011.

25. Cannon RM,LeGrand R, Chagpar RB, Ahmad SA, McClaine R, Kim HJ, Rupp C, Cho CS, Brinkman A, Weber S, et al: Multi-institutional analysis of pancreatic adenocarcinoma demonstrating the effect of diabetes status on survival after resection. HPB Oxf 14: 228-235, 2012

26. van de Poll-Franse LV, Houterman S, Janssen-Heijnen ML, Dercksen MW, Coebergh JW and Haak HR: Less aggressive treatment and worse overall survival in cancer patients with diabetes: a large population based analysis. Int J Cancer 120: 1986-1992, 2007

27. Mao Y, Tao M, Jia X, Xu H, Chen K, Tang H and Li D: Effect of diabetes mellitus on survival in patients with pancreatic cancer: a systematic review and meta-analysis. Sci Rep 5: $17102,2015$.

28. Elisei R, Lorusso L, Romei C, Bottici V, Mazzeo S, Giani C, Fiore E, Torregrossa L, Insilla AC, Basolo F, et al: Medullary thyroid cancer secreting carbohydrate antigen 19-9 (Ca 19-9): a fatal case report. J Clin Endocrinol Metab 98: 35503554, 2013

29. Pall M, Iqbal J, Singh SK and Rana SV: CA 19-9 as a serum marker in urothelial carcinoma. Urol Ann 4: 98-101, 2012.

30. Biadgo B and Abebe M: Type 2 diabetes mellitus and its association with the risk of pancreatic carcinogenesis: a review. Korean J Gastroenterol 67: 168-177, 2016.

31. Guillaumond F, Leca J, Olivares O, Lavaut MN, Vidal N, Berthezène $\mathrm{P}$, Dusetti NJ, Loncle $\mathrm{C}$, Calvo $\mathrm{E}$, Turrini $\mathrm{O}$, et al: Strengthened glycolysis under hypoxia supports tumor symbiosis and hexosamine biosynthesis in pancreatic adenocarcinoma. Proc Natl Acad Sci USA 110: 3919-3924, 2013.

32. Rajeshkumar NV, Dutta P, Yabuuchi S, de Wilde RF, Martinez GV, Le A, Kamphorst JJ, Rabinowitz JD, Jain SK, Hidalgo M, et al: Therapeutic targeting of the Warburg effect in pancreatic cancer relies on an absence of p53 function. Cancer Res 75: 3355-3364, 2015. 